\title{
Electrochemical Characterisation Study of Coatings Obtained by High Velocity Oxy-Fuel Spraying (HVOF)
}

\author{
P.H. Suegama, C.S. Fugivara, A.V. Benedetti", \\ J. Delgado ${ }^{\text {a) }}$, J.M. Guilemany ${ }^{\text {a) }}$ \\ Dep. Físico-Química, Instituto de Química, Universidade Estadual Paulista, UNESP. CP 355, \\ 14801-970 Araraquara, SP, Brazil.E-mail:benedeti@iq.unesp.br. \\ ${ }^{a)}$ CPT Thermal Spray Center. Materials Engineering. Dept. Enginyeria Química i Metallúrgia. \\ Universitat de Barcelona, C/Martí i Franquès 1. E-08028 Barcelona, Spain. \\ E-mail:cpt@material.qui.ub.es
}

Received 10 September 2002; accepted in revised form 5 February 2003

\begin{abstract}
The electrochemical behaviour of coated $\mathrm{Cr}_{3} \mathrm{C}_{2}-\mathrm{NiCr}$ steel in aerated $0.5 \mathrm{M} \mathrm{H}_{2} \mathrm{SO}_{4}$ solution was studied by means of electrochemical ac and dc measurements. The structural characterisation of the coated steel, before and after electrochemical tests, was also performed in order to identify the mechanism of the electrolyte penetration through the coating up to the steel substrate, causing its corrosion. This characterisation may also help to explain electrochemical results.

Three types of $\mathrm{Cr}_{3} \mathrm{C}_{2}-\mathrm{NiCr}$ coatings performed by a High Velocity Oxy-Fuel Spraying system (HVOF) were analysed. The facility for the electrolyte penetration through the coating and the corresponding electrochemical behaviour of the samples were strongly influenced by the spray parameters used and heat treatment applied. It was observed that heat-treated coatings (Q1 and Q3) showed better corrosion resistance than the assprayed coating (A). For coatings Q1 and Q3, the electrolyte did not reach the steel substrate during the measurement, leading to a better protection of the steel substrate against corrosion.
\end{abstract}

Keywords: corrosion, $\mathrm{Cr}_{3} \mathrm{C}_{2}-\mathrm{NiCr}$ coating, electrochemical techniques, $\mathrm{HVOF}$, thermal spraying.

\section{Introdução}

Inicialmente, a projeção térmica foi basicamente desenhada para a obtenção de revestimentos resistentes ao desgaste e à abrasão [1,2]. $\mathrm{O}$ aumento na utilização dos revestimentos em meios cada vez mais corrosivos tornou necessário seu

\footnotetext{
* Corresponding author. E-mail address: benedeti@iq.unesp.br. Communication presented at XV IberoAmerican Meeting of Electrochemistry.
} 
estudo frente aos mecanismos de corrosão. As características intrínsecas da projeção térmica [3] como sua versatilidade, possibilidade de aplicação desde polímeros até cerâmicas e a categoria de equipamentos de projeção já existentes no mercado, fazem desta, uma técnica fundamental e de grande expansão na obtenção e reparo de qualquer tipo de peça industrial [4].

Revestimentos obtidos por técnicas de projeção térmica são muito aplicados industrialmente, devido à possibilidade de aplicação sobre diferentes materiais, desde polímeros a cerâmicas, a um custo relativamente baixo, com rápida e fácil aplicação sem restrições de tamanho e com boa reprodutibilidade $[1,5]$.

A técnica de HVOF apresenta as vantagens de ter maior plasticidade, fluidez e velocidade do material projetado, permitindo que as partículas alcancem o substrato, no momento do impacto, adquirindo uma forma lenticular mediante a união perfeita de junção destas lentículas. Isto evolui até alcançar uma morfologia de um bom revestimento, com elevada densidade, baixa porosidade e boa aderência, etc. $[1,6]$.

A qualidade do revestimento dependerá de variáveis do processo, como a forma, tamanho e distribuição das partículas de pó; da proporção dos gases combustíveis, aditivos, portadores e da velocidade relativa com que se desloca a pistola de projeção-substrato [1,8]. Estas são variáveis de maior influência na qualidade do revestimento final e, como conseqüência, responsáveis pela estrutura do material obtido [1].

Os revestimentos obtidos por HVOF contêm tensões residuais resultantes da contração durante o resfriamento e a solidificação. A grandeza desta tensão varia, dependendo dos parâmetros usados no processo durante a projeção do revestimento [9]. O material revestido rachará se a magnitude da tensão residual exceder sua força de adesão ao substrato. Métodos utilizados para reduzir esta tensão incluem: expansão do substrato por um pré-aquecimento, seleção do material a ser projetado, introdução de camadas intermediárias e a macro rugosidade da superfície do substrato.

Neste trabalho foi estudado o efeito do tratamento térmico com a chama da pistola sobre a resistência do revestimento à corrosão em $\mathrm{H}_{2} \mathrm{SO}_{4} 0.5 \mathrm{M}$. 


\section{Procedimento Experimental}

O substrato utilizado foi um aço 42CrMo6 jateado com alumina, cuja composição está descrita na Tabela 1.

Tabela 1. Composição do aço (\% em massa) utilizado como substrato.

\begin{tabular}{ccccccc}
\hline Aço & UNS & C & Si & Mn & P & S \\
\hline 42CrMo6 & G11200 & 0.22 & 0.28 & 0.51 & 0.02 & 0.02 \\
\hline
\end{tabular}

Um pó comercial Amdry - 5420 de composição nominal $75 \% \mathrm{Cr}_{3} \mathrm{C}_{2}-25 \% \mathrm{NiCr}$ com densidade de $5.91 \mathrm{~g} \mathrm{~cm}^{-3}$ e diâmetro de partícula de $37.15 \mu \mathrm{m}$ foi projetado com uma pistola Sulzer Metco CDS PT-100 utilizando os parâmetros de projeção mostrados na Tabela 2.

Uma mistura dos gases propileno e oxigênio foi utilizada para a projeção. $\mathrm{O}$ revestimento A foi obtido com parâmetros padrões e os revestimentos Q1 e Q3 foram obtidos utilizando uma chama mais energética que produz estrutura mais densa, com menos trincas e poros [10].

Dois tipos de revestimentos A e Q1 foram projetados utilizando a mesma velocidade de deslocamento da pistola, $500 \mathrm{~mm} \mathrm{~s}^{-1}$, e o revestimento Q3 com o dobro da velocidade, $1000 \mathrm{~mm} \mathrm{~s}^{-1}$. Os revestimentos Q1 e Q3 foram tratados termicamente (Tabela 3) durante a formação do revestimento utilizando a chama da pistola (sem pó) imediatamente após as primeiras camadas.

A célula eletroquímica utilizada era composta por um eletrodo de referência de $\mathrm{Hg} / \mathrm{Hg}_{2} \mathrm{SO}_{4}, \mathrm{~K}_{2} \mathrm{SO}_{4}$ saturado conectado a solução por um capilar de LugginHaber, um eletrodo auxiliar (rede de platina) e o quarto eletrodo que era um fio de platina ( $0.2 \mathrm{~mm}$ de diâmetro) ligado ao eletrodo de referência por meio de um capacitor de $10 \mu \mathrm{F}$. A área geométrica do eletrodo de trabalho exposta à solução era de $0.33 \mathrm{~cm}^{2}$. 
Tabela 2. Condições de preparo dos revestimentos.

\begin{tabular}{lcccccc}
\hline Amostra & $\begin{array}{c}\mathrm{O}_{2} \\
\mathrm{~L} \mathrm{~min}^{-1}\end{array}$ & $\begin{array}{c}\mathrm{C}_{3} \mathrm{H}_{6} \\
\mathrm{~L} \mathrm{~min}^{-1}\end{array}$ & $\begin{array}{c}\mathrm{n}^{0} \mathrm{de} \\
\text { camadas }\end{array}$ & $\begin{array}{c}\mathrm{d}_{\text {proj }}{ }^{*} \\
\mathrm{~mm}\end{array}$ & $\begin{array}{c}\mathrm{v}^{* *} \\
\mathrm{~mm} / \mathrm{s}\end{array}$ & Trat. térmico \\
\hline A & 420 & 60 & 15 & 300 & 500 & Não \\
Q1 & 483 & 69 & 15 & 200 & 500 & Sim \\
Q3 & 483 & 69 & 30 & 200 & 1000 & Sim \\
\hline
\end{tabular}

*Distância de projeção, ${ }^{* *}$ velocidade de deslocamento da pistola.

Tabela 3. Condições do tratamento térmico.

\begin{tabular}{lccccccc}
\hline Amostra & $\begin{array}{c}\mathrm{n}^{0} \text { de camadas } \\
\text { iniciais }\end{array}$ & $\begin{array}{c}\mathrm{O}_{2} \\
\mathrm{~L} \mathrm{~min}^{-1}\end{array}$ & $\begin{array}{c}\mathrm{C}_{3} \mathrm{H}_{6} \\
\mathrm{~L} \mathrm{~min}^{-1}\end{array}$ & $\begin{array}{c}\mathrm{d}_{\text {proj }} \\
\mathrm{mm}\end{array}$ & $\begin{array}{c}\mathrm{v}^{* *} \\
\mathrm{~mm} / \mathrm{s}\end{array}$ & $\begin{array}{c}\mathrm{n}^{0} \text { de camadas } \\
\text { finais }\end{array}$ \\
\hline Q1 & 5 & 483 & 69 & 105 & 500 & 10 & \\
Q3 & 10 & 483 & 69 & 105 & 1000 & 20 \\
\hline
\end{tabular}

Os experimentos foram feitos utilizando $80 \mathrm{~mL}$ de solução de $\mathrm{H}_{2} \mathrm{SO}_{4} 0.5 \mathrm{M}$ saturada em ar e não agitada. As medidas de potencial de circuito aberto foram realizadas no potenciostato-galvanostato EG\&G/PAR, modelo 273A. As medidas de espectroscopia de impedância eletroquímica (EIE) foram feitas utilizando um analisador de freqüência Solartron modelo 1255.

As medidas de EIE foram realizadas aplicando o potencial de circuito aberto, medido após estabilização do potencial do eletrodo em solução. O intervalo de freqüências foi $50 \mathrm{kHz}$ a $10 \mathrm{mHz}$, com amplitude de $\pm 5 \mathrm{mV}$ rms e 7 pontos por década. Os dados de impedância eletroquímica foram tratados com o método dos mínimos quadrados não lineares (programa Boukamp), empregando circuito equivalente, onde se buscou obter os mínimos valores para $\chi^{2}$, o circuito mais simples e com significado físico considerando-se a interface eletrodo/solução em estudo.

As curvas de polarização foram obtidas após estabilização do potencial em circuito aberto. A velocidade de varredura foi $0.166 \mathrm{mV} \mathrm{s}^{-1}$, e os intervalos de potenciais foram de $\eta=-0.100 \mathrm{mV}$ a $\eta=+0.350 \mathrm{mV}$, relativo ao potencial medido em circuito aberto após sua establização. Todas as medidas foram realizadas mantendo-se a temperatura a $25{ }^{0} \mathrm{C}$. 
A caracterização estrutural dos revestimentos foi feita por microscopia óptica e eletrônica. Para a análise foram utilizados: microscópio óptico da Leica DMR com analisador de imagens controlado pelo Software Leica Qwin para a determinação da porosidade e um microscópio eletrônico de varredura TOPCON SM300 acoplado a um espectrômetro de energia dispersiva (EDS).

\section{Resultados e Discussão}

\section{Caracterização antes e depois dos testes}

Os revestimentos obtidos apresentaram uma estrutura homogênea com porosidade ao redor de $1 \%$ e espessura de $253 \mu \mathrm{m}$ para o revestimento obtido sob as condições de projeção A, $306 \mu \mathrm{m}$ para Q1 e $350 \mu \mathrm{m}$ para Q3.

A Fig. 1 mostra a secção transversal do revestimento $\mathrm{A}$, tendo indicados seus vários componentes. Uma análise detalhada desse revestimento [11] mostrou que o sistema é composto por uma matriz nanocristalina-NiCr (A) com diferentes porcentagens de $\mathrm{Cr}\left(20-50 \%\right.$ em massa); carbetos de cromo, como $\mathrm{Cr}_{3} \mathrm{C}_{2}$ (B) e $\mathrm{Cr}_{7} \mathrm{C}_{3}$ ou $\mathrm{Cr}_{23} \mathrm{C}_{6}$, que correspondem à decomposição de $\mathrm{Cr}_{3} \mathrm{C}_{2}$ durante a projeção; pequena quantidade de $\mathrm{Cr}_{2} \mathrm{O}_{3}$ (C); poros (D) e microtrincas (E) entre as diferentes camadas depositadas.

A caracterização estrutural do revestimento A antes do teste de corrosão apresentou mais poros e trincas. Os revestimentos Q1 e Q3 apresentaram as mesmas características que o revestimento A na parte inferior, ou seja, antes do tratamento térmico feito com a chama da pistola. Depois do tratamento térmico apresentaram menos poros e menos trincas (Figs. 1 e 2).

Na Fig. 3a observa-se a seção transversal do revestimento A após imersão em solução de $\mathrm{H}_{2} \mathrm{SO}_{4} 0.5 \mathrm{M}$. A separação do revestimento/substrato ocorre quando a amostra é imersa no eletrólito por $\sim 40$ horas, e submetida a polarização de -0.1 a $0.35 \mathrm{~V} / \mathrm{E}_{\mathrm{CA}}$, a $0.16 \mathrm{mVs}^{-1}$. Isto ocorre devido ao revestimento A possuir uma estrutura mais aberta, que facilita a penetração do eletrólito no revestimento. Se o eletrólito atinge o substrato, o processo de dissolução se inicia, uma vez que o substrato é menos nobre que o revestimento. Já nos revestimentos tratados termicamente, o eletrólito não separa o substrato do revestimento (Fig. 3b e 3c). 


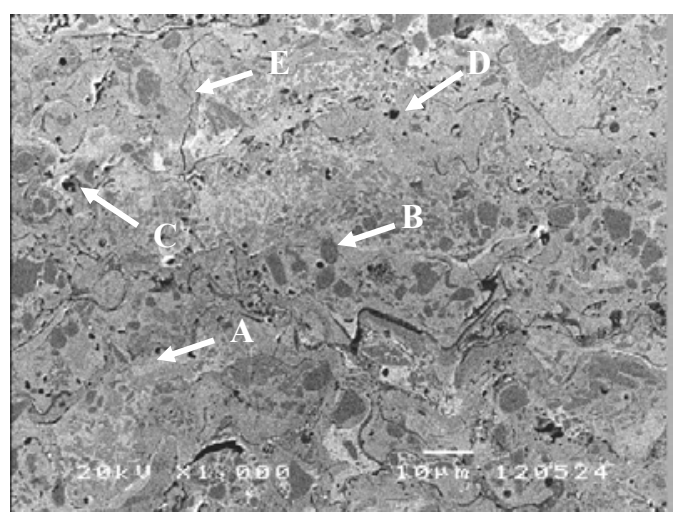

Figura 1. Imagem SEM da secção transversal do revestimento A.

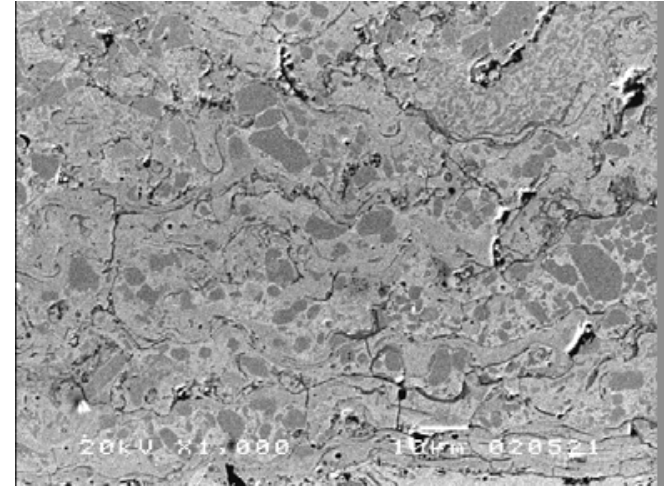

(a)

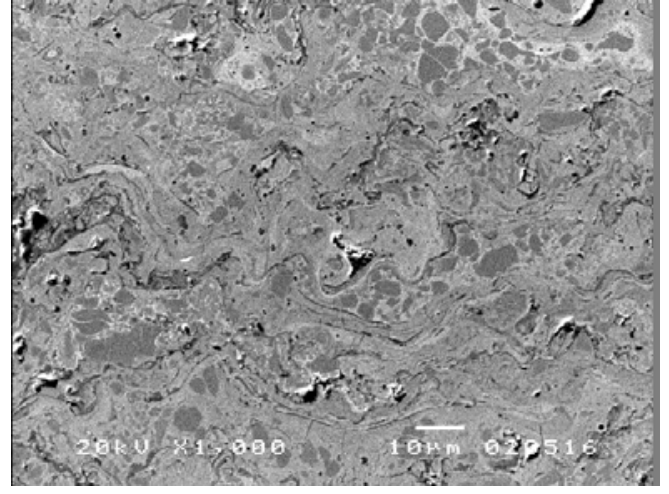

(b)

Figura 2. Imagem SEM da secção transversal do revestimento Q1: (a) camadas antes do tratamento térmico e (b) camadas após o tratamento térmico.

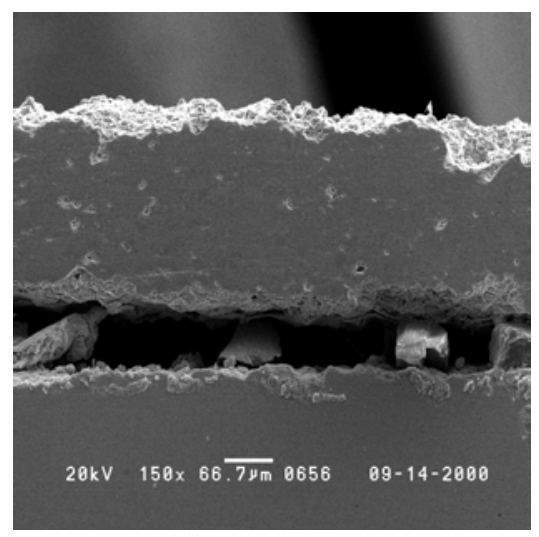

(a)

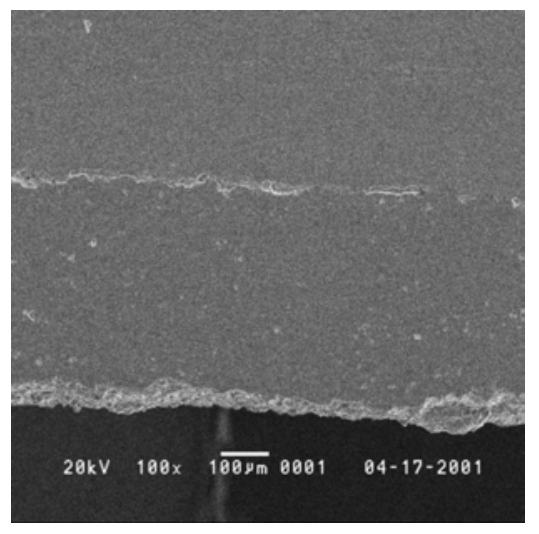

(b) 


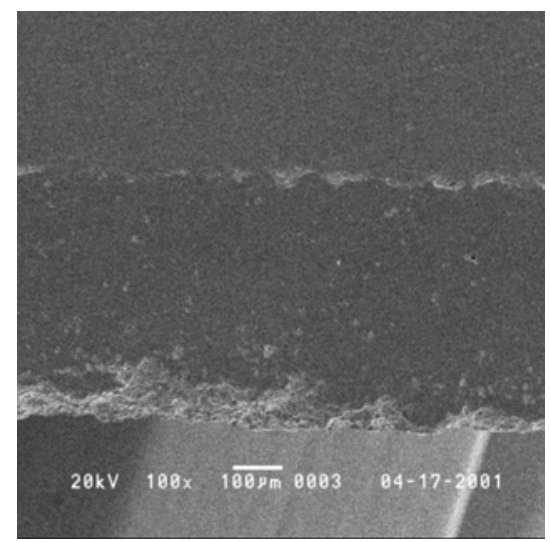

(c)

Figura 3. Imagem SEM da secção transversal após imersão no eletrólito após $\sim 40 \mathrm{~h}$ do: (a) revestimento $\mathrm{A}$, (b) revestimento Q1 e (c) revestimento Q3.

\section{Caracterização eletroquímica}

Medidas de potencial de circuito aberto em função do tempo

A Fig. 4 mostra curvas de potencial de circuito aberto em função do tempo para os revestimentos $\mathrm{A}, \mathrm{Q} 1$ e $\mathrm{Q} 3\left(\mathrm{E}_{\mathrm{CA}}=-0.84 \mathrm{~V},-0.76 \mathrm{~V}\right.$ e $-0.73 \mathrm{~V}$, respectivamente) durante 6 horas de imersão em solução de $\mathrm{H}_{2} \mathrm{SO}_{4} 0.5 \mathrm{M}$. Para o revestimento A, a curva de $\mathrm{E}_{\mathrm{CA}}$ versus tempo mostra uma diminuição brusca do potencial durante a primeira hora de imersão. O revestimento A é menos espesso que os demais, é mais poroso e apresenta maior número de trincas, por isso o eletrólito penetra mais facilmente até atingir o substrato. Portanto, em pouco tempo o potencial alcançou valor similar àquele encontrado no substrato sem o revestimento, resultando numa diminuição brusca do potencial desde seu valor na interface revestimento/eletrólito para o da interface substrato/eletrólito. $\mathrm{Na}$ interface revestimento/aço forma-se um par galvânico que acelera o ataque ao aço, levando à separação do revestimento do substrato. Isto ocorre pois, há um aumento da acidez local, dissolvendo os óxidos-hidróxidos formados na base dos poros e trincas, expondo assim partes do aço que estão praticamente livres de produtos de corrosão. No caso dos revestimentos Q1 e Q3, ambos sofreram um tratamento térmico com chama da pistola após a aplicação de, respectivamente, 5 e 10 camadas, ou seja, aproximadamente $100 \mu \mathrm{m}$. Esse tratamento térmico permitiu melhor acomodação das camadas subseqüentes, produzindo um revestimento com menor porosidade e oferecendo uma espécie de barreira à penetração do 
eletrólito até o substrato. Nestes casos, o potencial de circuito aberto diminuiu na primeira hora de imersão para valores menos negativos que para o revestimento A, porque o eletrólito não penetrou até o substrato. A diminuição inicial do potencial da interface revestimento/eletrólito pode estar associada à dissolução de óxidos da superfície e do interior do revestimento.

A amostra A é mais fina que as demais, porém, mesmo que fosse mais espessa ou de igual espessura, a penetração do eletrólito ocorreria da mesma forma, já que os poros e trincas neste tipo de amostra são grandes. Os parâmetros de obtenção deste revestimento são os sugeridos pelo fabricante do pó como condições standard.

O potencial de estabilização dos revestimentos Q1 e Q3 são mais elevados, indicando que as condições de projeção e o tratamento térmico aplicado a estes revestimentos produziram uma maior proteção do substrato ao ataque ácido. $\mathrm{O}$ revestimento Q1 leva mais tempo para estabilizar o potencial provavelmente devido a maior diferença entre a superfície da amostra e seu interior, especialmente até a região onde foi feito o tratamento térmico com a pistola, uma vez que cada camada tem espessura de ca. $20 \mu \mathrm{m}$. A amostra Q3 é formada por uma somatória de camadas mais finas (ca. $10 \mu \mathrm{m}$ cada) e formou-se com uma distribuição de temperatura das partículas mais homogênea porque foi preparada com velocidade de projeção duas vezes maior, além de ter sofrido o mesmo tratamento térmico da amostra Q1. Isso deve explicar o maior potencial de estabilização dessa amostra, como também as diferenças observadas em outras medidas eletroquímicas.

\section{Medidas de Espectroscopia de Impedância Eletroquímica (EIE)}

Após estabilizar o potencial de circuito aberto foram realizadas medidas de impedância eletroquímica. Para tratar os dados obtidos por meio das medidas de EIE, diversos circuitos equivalentes foram experimentados por meio do programa desenvolvido por Boukamp. O circuito elétrico equivalente que forneceu melhor ajuste nos dados obtidos (Fig. 5), menores erros relativos de cada parâmetro e menor $\chi^{2}$ (Tabela 4), foi o circuito $\mathrm{R}_{\mathrm{s}}\left(\mathrm{R}_{1} \mathrm{Q}_{1}\right)\left(\mathrm{R}_{2} \mathrm{Q}_{2}\right)$, (Fig. 6). 


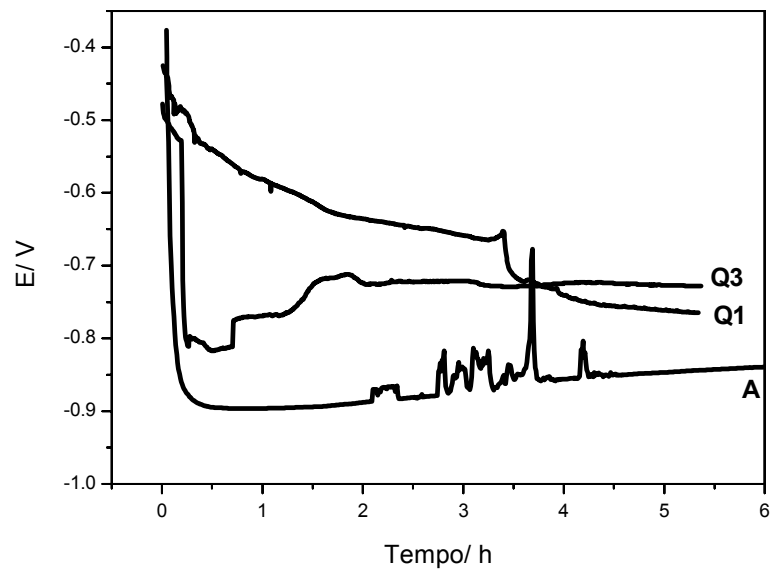

Figura 4. Gráfico do $\mathrm{E}_{\mathrm{CA}}$ em função do tempo para as amostras revestidas nas condições A, Q1 e Q3 em solução de $\mathrm{H}_{2} \mathrm{SO}_{4} 0.5 \mathrm{M}$, saturada em ar e não agitada.

Para o revestimento $A$, neste circuito, $R_{s}$ é a resistência não compensada da solução entre o capilar de Luggin do eletrodo de referência e a superfície do eletrodo de trabalho. Os valores de $R_{s}$ permaneceram praticamente constantes para todas as amostras. $\mathrm{R}_{1}$ e $\mathrm{Q}_{1}$ estão relacionados com a resistência do eletrólito no interior dos poros do revestimento e a capacitância do próprio revestimento e $\mathrm{R}_{2}$ e $\mathrm{Q}_{2}$ com a resistência de transferência de carga para a oxidação do substrato e a capacitância da interfase revestimento/eletrólito e substrato livre de revestimento (poros-trincas/eletrólito), $\mathrm{Y}_{0.2}$, devendo ser portanto elevada em função da espessura do revestimento.

No caso dos revestimentos Q1 e Q3, $R_{1}$ e $R_{2}$ estão relacionadas com o revestimento, pois o eletrólito não alcançou o substrato [1]. As micrografias da Fig. 3 mostram que mesmo após 48 h de imersão, o eletrólito ainda não atingiu o substrato, enquanto que o revestimento A já se destacou do substrato. Nestes revestimentos se têm óxidos de níquel e a matriz de $\mathrm{NiCr}$ que podem estar sendo atacados pelo eletrólito. $\mathrm{O}$ eletrólito penetra no revestimento atacando, provavelmente os óxidos de níquel e a matriz de $\mathrm{NiCr}$ contidos ao longo do revestimento. Portanto, $\mathrm{Y}_{0.1}$ estaria relacionada com a capacitância do revestimento e, $\mathrm{Y}_{0.2}$ com a capacitância da interfase revestimento (incluindo a solução no interior de trincas, poros)/eletrólito. 
Os valores de $\mathrm{n}_{1}$ obtidos para todos revestimentos, $c a$. 0.5 , são típicos de um eletrodo poroso [12-16]. Já um valor de n próximo a 1 é esperado para superfícies homogêneas e lisas [13] e ao redor de 0.7 pode indicar uma superfície heterogênea ou rugosa.

Os resultados das Tabelas 4 e 5 indicam que as amostras tratadas termicamente apresentam maior resistência, tanto para $R_{1}$ como para $R_{2}$, protegendo melhor $o$ aço contra a ação do eletrólito.

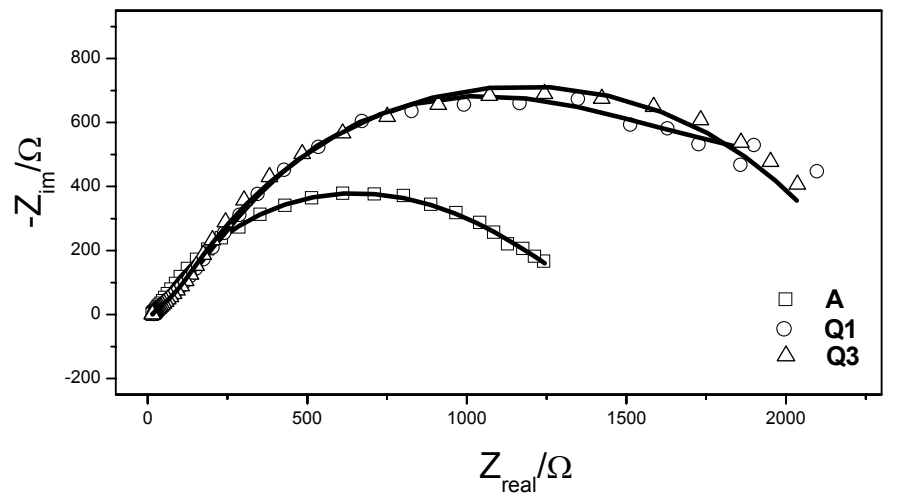

Figura 5. Diagramas de impedância experimental (o) e ajustado (-) para os revestimentos estudados aplicando $\mathrm{E}_{\mathrm{CA}}$ em solução de $\mathrm{H}_{2} \mathrm{SO}_{4} 0.5 \mathrm{M}$ saturada em ar e não agitada.

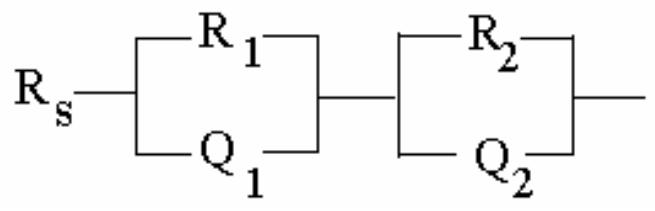

Figura 6. Circuito elétrico equivalente que ajustou os dados obtidos para os revestimentos $\mathrm{A}, \mathrm{Q} 1$ e Q3 em solução de $\mathrm{H}_{2} \mathrm{SO}_{4} \quad 0.5 \mathrm{M}$ saturada em ar e não agitada aplicando o $\mathrm{E}_{\mathrm{CA}}$.

Curvas de polarização

Estas curvas são analisadas somente qualitativamente devido a natureza complexa dos revestimentos, não fazendo sentido utilizar a equação de ButlerVolmer para estimar os parâmetros de Tafel. 
Tabela 4. Valores obtidos pelo tratamento dos dados de impedância eletroquímica para os revestimentos após $20 \mathrm{~h}$ em solução de $\mathrm{H}_{2} \mathrm{SO}_{4} 0.5 \mathrm{M}$ saturada em ar e não agitada.

\begin{tabular}{cccc}
\hline Revestimento & $\mathbf{Q 3}$ & $\mathbf{Q 1}$ & $\mathbf{A}$ \\
\hline $\mathbf{R}_{\mathbf{s}} / \Omega$ & 13 & 18 & 14 \\
$\mathbf{\%}$ & 1.9 & 1.3 & 0.25 \\
$\mathbf{R}_{\mathbf{1}} / \mathbf{k} \Omega$ & 1.6 & 0.11 & 0.66 \\
$\mathbf{\%}$ & 10 & 15 & 3.5 \\
$\mathbf{Y}_{\mathbf{0 . 1}} / \mathbf{S} \mathbf{s}^{\mathbf{n}}$ & $1.4 \times 10^{-3}$ & $9.7 \times 10^{-4}$ & $2.4 \times 10^{-3}$ \\
$\mathbf{\%}$ & 6.8 & 9.5 & 1.6 \\
$\mathbf{n}_{\mathbf{1}}$ & 0.47 & 0.50 & 0.50 \\
$\mathbf{\%}$ & 2.1 & 2.6 & $\mathrm{FIXO}$ \\
$\mathbf{R}_{\mathbf{2}} / \mathbf{k} \Omega$ & 0.77 & 2.0 & 0.77 \\
$\mathbf{\%}$ & 13 & 2.0 & 1.9 \\
$\mathbf{Y}_{\mathbf{0 . 2}} / \mathbf{S} \mathbf{s}^{\mathbf{n}}$ & $1.5 \times 10^{-3}$ & $5.4 \times 10^{-4}$ & $8.6 \times 10^{-4}$ \\
$\mathbf{\%}$ & 8.0 & 1.5 & 0.59 \\
$\mathbf{\mathbf { n } _ { 2 }}$ & 0.97 & 0.74 & 0.76 \\
$\mathbf{\%}$ & 4.3 & 1.4 & 0.53 \\
$\chi^{\mathbf{2}}$ & $1.0 \times 10^{-3}$ & $2.9 \times 10^{-4}$ & $4.0 \times 10^{-5}$ \\
\hline
\end{tabular}

Tabela 5. Valores obtidos pelo tratamento dos dados de impedância eletroquímica para os revestimentos após $\sim 48 \mathrm{~h}$ em solução de $\mathrm{H}_{2} \mathrm{SO}_{4} 0.5 \mathrm{M}$ saturada em ar e não agitada.

\begin{tabular}{cccc}
\hline Revestimento & $\mathbf{Q 3}$ & $\mathbf{Q 1}$ & $\mathbf{A}$ \\
\hline $\mathbf{R}_{\mathbf{s}} / \Omega$ & 13 & 18 & 13 \\
$\mathbf{\%}$ & 2.7 & 1.5 & 0.27 \\
$\mathbf{R}_{\mathbf{1}} / \mathbf{k} \Omega$ & 4.2 & 0.137 & 0.160 \\
$\mathbf{\%}$ & 22 & 21 & 16 \\
$\mathbf{Y}_{\mathbf{0 . 1}} / \mathbf{S} \mathbf{s}^{\mathbf{n}}$ & $1.6 \times 10^{-3}$ & $1.0 \times 10^{-3}$ & $4.1 \times 10^{-3}$ \\
$\mathbf{\%}$ & 8.1 & 9.9 & 3.8 \\
$\mathbf{n}_{\mathbf{1}}$ & 0.45 & 0.50 & 0.50 \\
$\mathbf{\%}$ & 2.7 & 2.8 & $\mathrm{FIXO}$ \\
$\mathbf{R}_{\mathbf{2}} / \mathbf{k} \boldsymbol{\Omega}$ & 1.3 & 1.8 & 0.58 \\
$\mathbf{\%}$ & 12 & 3.4 & 3.3 \\
$\mathbf{Y}_{\mathbf{0 . 2}} / \mathbf{S} \mathbf{s}^{\mathbf{n}}$ & $1.4 \times 10^{-3}$ & $5.5 \times 10^{-4}$ & $7.6 \times 10^{-4}$ \\
$\mathbf{\%}$ & 7.6 & 2.4 & 1.1 \\
$\mathbf{n}_{\mathbf{2}}$ & 0.98 & 0.77 & 0.74 \\
$\mathbf{\%}$ & 4.0 & 2.2 & 0.55 \\
$\chi^{\mathbf{2}}$ & $1.8 \times 10^{-3}$ & $4.4 \times 10^{-4}$ & $4.3 \times 10^{-5}$ \\
\hline
\end{tabular}




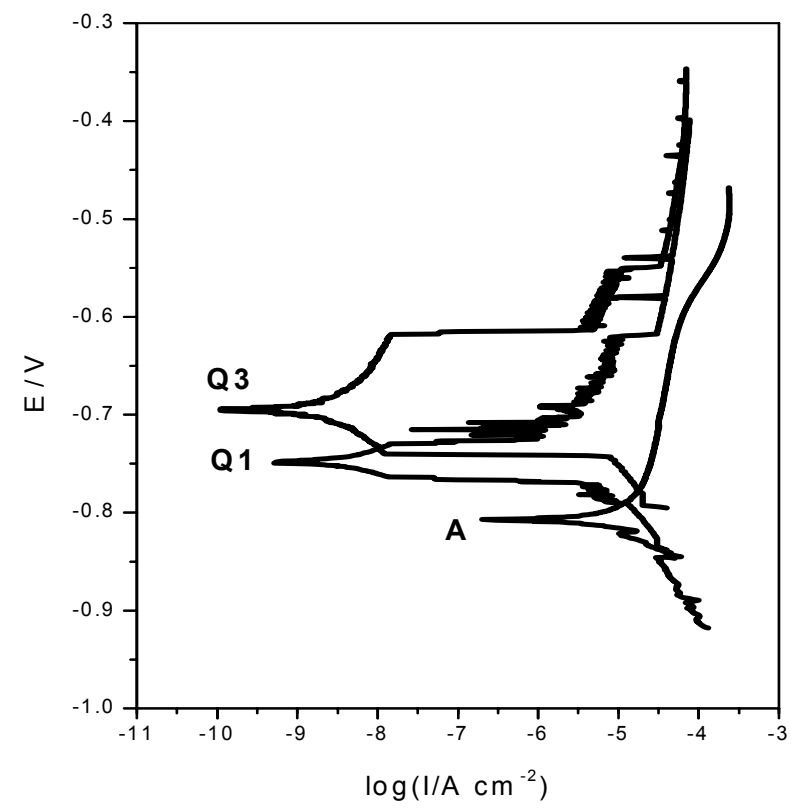

Figura 7. Curvas de polarização para as amostras A, Q1 e Q3 obtidas em solução de $\mathrm{H}_{2} \mathrm{SO}_{4} 0.5 \mathrm{M}$ saturada em ar e não agitada, $\mathrm{v}=0.166 \mathrm{mV} \mathrm{s}^{-1}$.

As curvas de polarização obtidas para os revestimentos, após estabilização do potencial na solução de ácido sulfúrico, encontram-se na Fig. 7. Os ramos catódico e anódico dessas curvas foram obtidos sobre a mesma área da amostra e no sentido de aumento do potencial. Portanto, é muito provável que haja contribuição da geração de hidrogênio, cujo desprendimento das micro-bolhas seria responsável pela oscilação da densidade de corrente observada no ramo catódico. As oscilações no ramo anódico podem estar associadas à formação e dissolução dos produtos de corrosão, uma vez que é observada na região de dissolução do substrato ou de oxidação do revestimento. Da observação das curvas de polarização percebe-se a maior inibição do processo de corrosão do substrato pela aplicação do revestimento Q3, seguida do Q1 por esta apresentar um maior $\mathrm{E}_{\mathrm{Corr}}$ e uma menor intensidade de corrente que as demais amostras obtidas em condições padrões A. 


\section{Conclusões}

Não se observou corrosão generalizada dos revestimentos de $\mathrm{Cr}_{3} \mathrm{C}_{2}-\mathrm{NiCr}$ no eletrólito testado.

O eletrólito penetra até o substrato por meio de poros, fendas e separações entre as camadas formadas durante o processo de projeção nos revestimentos obtidos em condições padrões A. Uma vez que o eletrólito alcança o substrato, este sofre corrosão preferencialmente devido ao par galvânico formado entre o substrato e o revestimento.

Os revestimentos tratados termicamente com a chama da pistola apresentam maiores resistências à corrosão. Os tratamentos térmicos retardam o efeito do eletrólito no substrato, formando "barreiras" que impedem a passagem do eletrólito ao longo do revestimento.

A amostra Q3, que combina maior fluxo dos gases, maior velocidade de deslocamento da pistola e tratamento térmico com a mesma chama da pistola, é a que apresenta maior resistência à corrosão do substrato.

\section{Agradecimentos}

Autores agradecem: FAPESP - Fundação de Amparo à Pesquisa do Estado de São Paulo (Proc. no. 00/01893-9), CNPq-Conselho Nacional de Desenvolvimento Científico e Tecnológico (Proc. no. 521569/95-8) e a Universidade de Barcelona.

\section{Referências}

1. J. Delgado, Fenomenología y caracterización de la resistencia a la corrosión electroquímica en diversos medios agresivos de recubrimientos obtenidos por proyección térmica. (2001) 515 p. Tese (Doutorado) - Universidade de Barcelona, Barcelona.

2. T. Kinos, S.L. Chen, P. Siitonen, P. Kettunen, International Thermal Spray Conference, 1995, Kobe p. 573-576.

3. J.M. Guilemany, J.R. Miguel, J.M. De Paco, Ciência e ingeniería de la superficie de los materiales metálicos (1996) cap. 18, p. 265-278. 
4. T. Kinos, S.L. Chen, P. Siitonen, P. Kettunen, Corrosion properties of shrouded plasma sprayed titanium coatings. International Thermal Spray Conference, 1995, Kobe p. 573-576.

5. P.L. Cabot, J. Fernandez, J.M. Guilemany, Mat. Sci. Forum 289-298 (1998) 667-678.

6. J. Guilemany, P. Cabot, J. Fernandez, J. Mat. Sci. Forum 289/292 (1998) $667-678$.

7. L. Pawlowsky, 'The Science and Engineering of Thermal Spray Coatings', John Wiley \& Sons Ed. (1995) 414 pp.

8. J. Hazan, C. Coddet, Mat. Sci. Forum 289-292 (1998) 719-728.

9. J.C. Tan, L. Looney, M.S.J. Hashmi, J. Mat. Proc. Tech. 92-93 (1999) 203208.

10. J. Guilemany, J. Fernández, J. Delgado, A.V. Benedetti, Influence of Thermal treatment in the corrosion behaviour of $\mathrm{HVOF} \mathrm{Cr}_{3} \mathrm{C}_{2}$ - $\mathrm{NiCr}$ coatings. ASM International Materials Park, (2001), p.1165-1169.

11.J.A. Calero, Caracterizacion de recubrimientos de Cermets de $\mathrm{Cr}_{3} \mathrm{C}_{2}-\mathrm{NiCr}$ obtenidos por HVOF e interpretación fenomenológica del processo mediante simulación matemática (1997) Tese (Doutorado) - Universidade de Barcelona, Barcelona.

12. R. De Levie, in: P. Delahay and W. Tobias (Eds.), Advances in Electrochemistry and Electrochemical Engineering, N.Y., (1967) p. 329.

13. M. Cai, S.M. Park, J. Electrochem. Soc. 143 (1996) 3895.

14.D.D. MacDonald, in R. Varma and J.R. Selman, Techniques for Characterization of Electrodes and Electrochemical Processes, John Wiley \& Sons, Inc. NY, (1991) p. 515.

15. R. De Levie, J. Electroanal. Chem. 281 (1990) 1.

16. S.G. Real, J.R. Vilche, A.J. Arvia, J. Electroanal. Chem. 341 (1992) 181. 